\title{
Biometric Template Classification: A Case Study in Iris Textures
}

\author{
Edara Srinivasa Reddy ${ }^{1}$, Chinnam SubbaRao ${ }^{2}$, and Inampudi Ramesh Babu ${ }^{3}$ \\ ${ }^{1,2}$ Research Scholar \\ ${ }^{3}$ Professor \\ Department of Computer Science, Acharya Nagarjuna Univerity, \\ Guntur, A.P, India \\ \{edara_67,rinampudi\}@yahoo.com
}

\begin{abstract}
Most of the biometric authentication systems store multiple templates per user to account for variations in biometric data. Therefore, these systems suffer from storage space and computation overheads. To overcome this problem the paper proposes techniques to automatically select prototype templates from iris textures. The paper has two phases: one is to find the feature vectors from iris textures that have less correlation and the second to calculate DU measure. Du measure is an effective measure of the similarity between two iris textures, because it takes into consideration three important perspectives: a) information, b) angle and e) energy. Also, gray level co occurrence matrix is used to find the homogeneity and correlation between the textures.
\end{abstract}

Keywords: Shaker iris, Jewel iris, Flower iris, Stream iris, gray level cooccurrence matrix, Spectral information divergence, Spectral angle mapper, DU measure.

\section{Introduction}

A typical iris biometric system operates in two distinct stages: the enrollment stage and the authentication stage [2]. During enrollment stage iris textures are acquired and processed to extract a feature set. The complex iris textures carry very distinctive information. The feature set includes some distinguished features like nodes and end point in the iris texture. The stored feature set, labeled with the user's identity, is referred as a template. In order to account for variations in the biometric based authentication system relies on the stability from an individual is susceptible to changes due to distance from the sensor, poor resolution of the sensor and alterations in iris texture due to cataract operations and others.

Multiple iris features pertaining to different portions of iris must be stored in data base. There is a trade off between the number of templates, and the storage and computational overheads introduced by multiple templates. An efficient system must in fact select the templates automatically.

\section{Basic Types of Iris Textures}

Depending on the texture of different human iris, we can group the irises into four basic groups. They are a) Stream iris, b) Jewel iris, c) Shaker iris and d) Flower iris. 
Also, there are different combinations of there groups. As a preliminary study we have concentrated to group the given data base into the above four classes.

a) Stream Iris: It contains a uniform fiber structure with subtle variations or streaks of color as shown in fig. 1.c. The structure of the iris is determined by the arrangement of the white fibers radiating from the center of the iris (or pupil). In this image one can notice that they are uniform and reasonably direct or parallel.

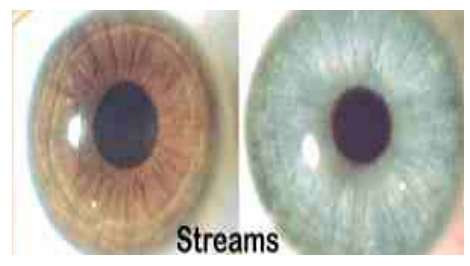

Fig. 1. a \& b. Stream Iris

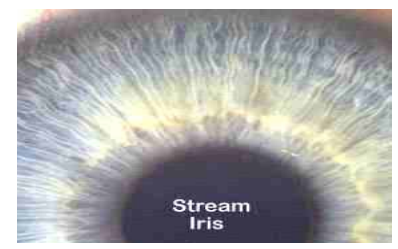

Fig. 1. c. Stream iris Texture

b) Jewel Iris: It contains dot-like pigments in the iris. The jewel iris can be recognized by the presence of pigmentation or colored dots on top of the fibers as shown in fig. 2.c. The dots (or jewels) can vary in color from light orange through black. They can also vary in size from tiny (invisible to the naked eye) to quite large.

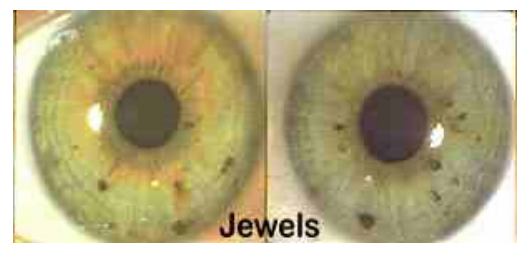

Fig. 2.a \& b. Jewel iris

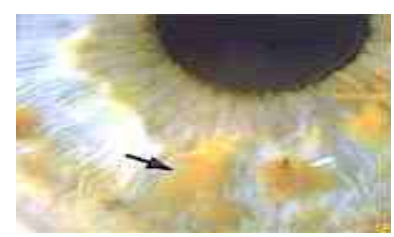

Fig. 2. c. Jewel iris Textures

c) Shaker iris: It contains dot-like pigments and rounded openings. The shaker iris is identified by the presence of both flower like petals in the fiber arrangement and pigment dots or jewels is shown in fig. 3.c. The presence of even one jewel in an otherwise Flower iris is sufficient to cause the occupant to exhibit shaker characteristics.

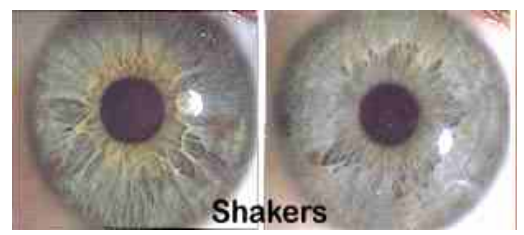

Fig. 3.a \& b. Shaker iris

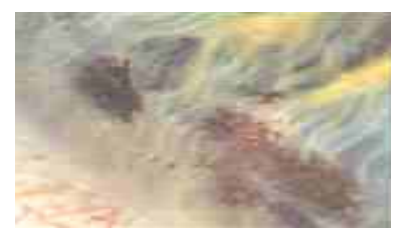

Fig. 3. c. Shaker iris textures

d) Flower iris: It contains distinctly curved or rounded openings in the iris. In a flower iris the fibers radiating from the center are distorted (in one or more places) to 
produce the effect of petals (hence the name flower) shown in fig. 4. In this image one can notice that they are neither regular nor uniform. A flower iris may have only one significant petal with the remainder of the iris looking like a stream

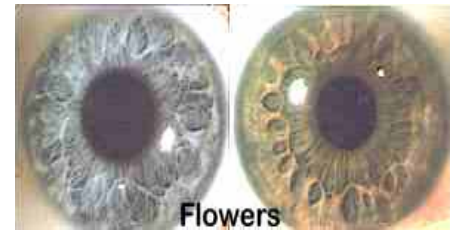

Fig. 4.a \& b. Flower iris

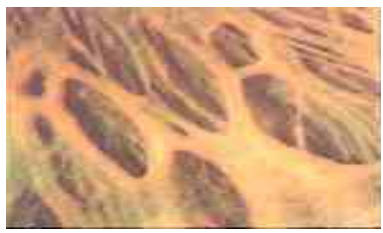

Fig. 4. c. Flower iris textures

\section{Template Selections and Updating}

The size of the same iris taken at different times may be different in the image as a result of changes in the camera to face distance. Due to simulation by light or for other reasons, such as hippos, natural continuous movement of the pupil, the pupil may be constricted or dilated [3]. The problem of template selection means, given a set of $\mathrm{N}$ iris images corresponding to a single human iris, selected $\mathrm{k}$ templates that best represent the similarity observed in $\mathrm{N}$ images. The selected templates must be updated time to time, since most of the users may under go cataract operations due to aging. To account for such changes, old templates must be replaced with newer ones. Some of the minutiae points like nodes and end points of iris textures may be added or deleted from the iris template. Thus template selection refers to the process by which iris textures are chosen from a given set of samples, where as template update refers to the process by which existing templates are replaced.

\section{Selection of Feature Vectors}

GLCM(gray-level co-occurrence matrix)[4], also known as the gray-level spatial dependence matrix, is a statistical measure used to characterize the texture of an image by calculating how often pairs of pixel, with specific values and in a specified spatial relationship occur in an image and then by extracting statistical measures from this matrix.

By default, the spatial relationship is defined as the pixel of interest and the pixel to its immediate right (horizontally adjacent), but one can specify other spatial relationships between the two pixels. Each element $(\mathrm{i}, \mathrm{j})$ in the resultant GLCM is simply the sum of the number of times that the pixel with value I occurred in the specified spatial relationship to a pixel with value $\mathrm{j}$ in the input image.

Initially, the dynamic range of the given image is scaled to reduce the number of intensity values in intensity image from 256 to 8 . The number of gray-levels, determine the size of the GLCM.

The gray-level co-occurrence matrix can reveal certain properties about the spatial distribution of the gray levels in the texture image. For example, if most of the entries 
in the GLCM are concentrated along the diagonal, the texture is coarse with respect to the specified offset.

However, a single GLCM might not be enough to describe the textual features of the input image. For example, a single horizontal offset might not be sensitive to texture with a vertical orientation. For this reason, gray co-matrix [5] can create multiple GLCMs for a single input image. TO create multiple GLCMs, specify an array of offsets to the gray co-matrix function. These offsets define pixel relationships of varying direction and distance. For, example, one can define an array of offsets that specify four directions (horizontal, vertical and two diagonals) and four distances. In this case, the input image is represented by 16 GLCMs. The statistics are calculated from these GLCMs and then the average is taken.

Statistical description of GLCM:

The statistical parameters that can be derived for GLCM are contrast, correlation, energy and homogeneity.

a) Contrast measures the local variations in the gray-level co-occurrence matrix.

b) Correlation measures the joint probability occurrence of the specified pixel pairs.

c) Energy provides the sum of squared elements in the GLCM.

Homogeneity measures the closeness of the distribution of elements in the GLCM to the GLCM diagonal.

We have derived only correlation to identify the length of the feature vector which has unique information from iris texture. A typical iris exhibits rich texture information in the immediate vicinity of the pupil which tapers away in intensity as one move away from the pupil. Thus, the iris templates are taken by segmenting a

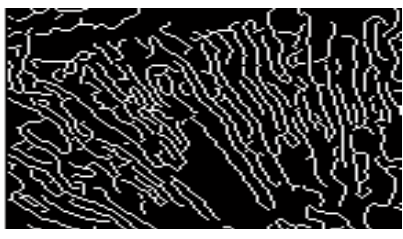

Fig:5a

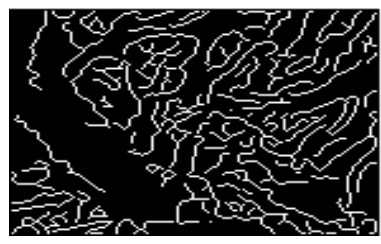

Fig:5c

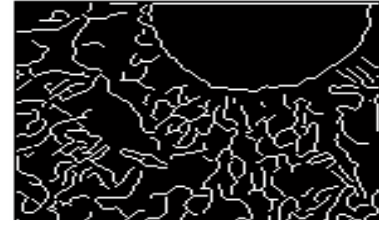

Fig:5b

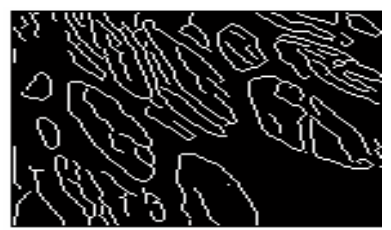

Fig:5d

Fig. 5. Iris textures after canny edge detection a)Stream iris b)Jewel iris c)Shaker iris d)Flower iris 
portion of iris texture near the pupil and from them the feature vectors are derived, by using correlation function. This is implemented by performing canny edge detection technique to produce binary image or respective iris texture and also by calculating the probability of occurrence of pixels.

The correlation Vs offset plot for different iris textures are given below.

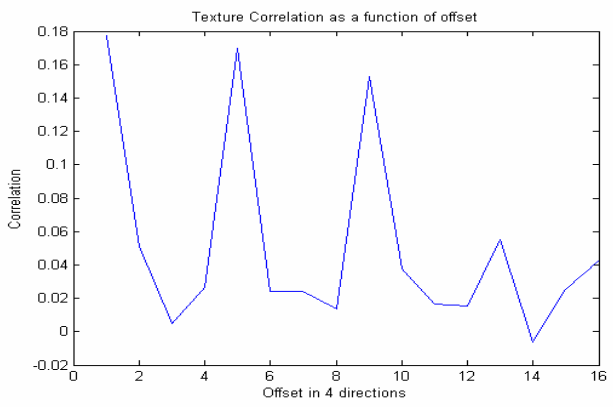

Fig. 6a. Correlation of Shaker iris texture

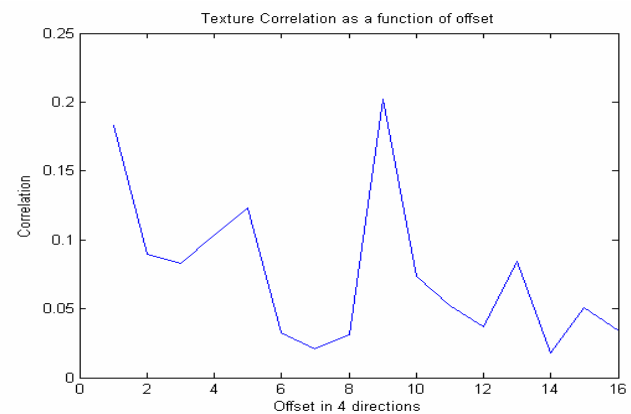

Fig. 6b. Correlation of Jewel iris texture

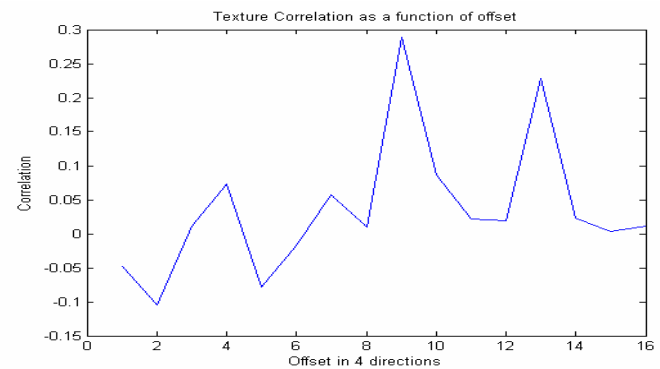

Fig. 6c. Correlation of Stream iris texture 


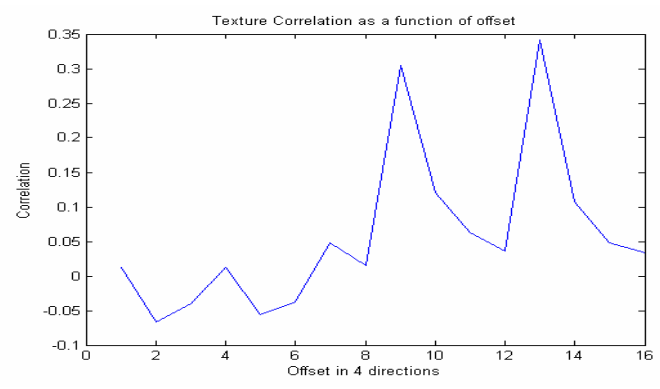

Fig. 6d. Correlation of Flower iris texture

From the above correlation plots, we can see the peak offsets which represent the periodic patterns that repeat for every set of pixels. Correlation function can be used to evaluate the coarseness. Larger texture primitives give rise to coarse texture and small primitives give fine texture. However if the primitives are periodic, then the correlation increases and decreases periodically with the distance [12]. For example, in the case of flower iris, the peak offsets are at 9 and 13 pixels. But, a common offset is observed at 9 for all textures. So, we can take a 9 pixel nearest neighbor as a feature vector to estimate DU measure in the case of iris textures.

\section{Du Measure}

$\mathrm{Du}$ measure[1] is an effective measure of the similarity between two iris textures, because it takes into consideration three important perspectives: a) information b) angle and c) energy.

a)Information: It is measured in terms of SID (spectral information divergence) between probability mass functions of the two textures $\mathrm{f}, \mathrm{g}$.

$\mathrm{SID}(\mathrm{f}, \mathrm{g})=\mathrm{D}(\mathrm{d} \| \mathrm{g})+\mathrm{D}(\mathrm{g} \| \mathrm{f})$

Here $D(f l l g)$ is the relative entropy of $g$ with respect to $f$.

Where $\mathrm{D}(\mathrm{f} \| \mathrm{g})=\Sigma(\mathrm{f} * \log (\mathrm{f} / \mathrm{g}))$ and $\mathrm{D}(\mathrm{g} \| \mathrm{f})=\Sigma(\mathrm{g} * \log (\mathrm{g} / \mathrm{f}))$

b) Angle: It is measured in terms of spectral angle mapper. It measures the angle between two vectors $f$ and $g$ given by,

$\operatorname{SAM}(\mathrm{f}, \mathrm{g})=\operatorname{acos}(<\mathrm{f}, \mathrm{g}>/(\|\mathrm{f}\| *\|\mathrm{~g}\|)$

Where $<\mathrm{f}, \mathrm{g}>=\Sigma \mathrm{f}_{\mathrm{i}}^{*} \mathrm{~g}_{\mathrm{i}}$, and $\|\mathrm{f}\|_{2}$ is the 2 -norm of vector $\mathrm{f},\|\mathrm{f}\|_{2}=<\mathrm{f}, \mathrm{f}>^{1 / 2}$

Mixed Measure:

$\mathrm{Du}$ et al[26] developed SID-SAM mixed measure. It is defined by a mixed measure, $M=\operatorname{SID}(f, g) * \tan (\operatorname{SAM}(f, g))$.

c) Energy: It is measured in terms of average power difference. Instead of using 2norm, we can use 1-norm. APD (average power difference) is given by,

$\operatorname{APD}(f, g)=\|f-g\| * 1 / N$, where $\|x\|_{1}=\Sigma|x|$.

Finally DU measure is given by,

$\mathrm{Du}(\mathrm{f}, \mathrm{g})=\mathrm{APD} *$ Mixed measure. 


\section{Iris Classification}

When a new iris texture is to be grouped into a predefined class, the Du measure is calculated with any one of the typical templates in that class. If the Du score is very high, we can say that it does not belong to that class. The new template belongs to a class, with which its Du measure is very low in comparison to others[1].

\section{Experimental Results}

We have taken for our study the iris image data base from CASIA iris image Data base[CAS03a] and MMU iris data base[MMU04a]. CASIA iris image data base contributes a total number of 756 iris images which were taken in two different time frames. Each of the iris images is 8-bit gray scale with resolution $320 \times 280$. MMU data base contributes a total number of 450 images which were captured by LG Iris Access $® 2200$.

Our studies conclude that all the iris images in the database have four different types of textures: Flower, Jewel, Stream and shaker. Thus there occur 6 different combinations for matching. Hence SID, SAM, APD values, for these 6 combinations, are given in the table.

Table 1. Du measures for different combinations of iris textures

\begin{tabular}{|l|l|l|l|l|l|}
\hline Combination & SID & SAM & MM & APD & DU \\
\hline Stream, Jewel & 1.51657 & 0.34578 & 0.54618 & 8.3483 & 4.55997 \\
\hline Stream, Flower & 1.60659 & 0.26289 & 0.43236 & 14.859 & 6.4244 \\
\hline Stream, Shaker & 1.8626 & 0.29232 & 0.56053 & 4.4424 & 2.4901 \\
\hline Flower, Jewel & 1.41048 & 0.40956 & 0.6123 & 6.491 & 3.9744 \\
\hline Flower, Shaker & 2.08906 & 0.27165 & 0.58188 & 10.397 & 6.0498 \\
\hline Jewel, Shaker & 2.14638 & 0.38986 & 0.88193 & 3.9059 & 3.447 \\
\hline
\end{tabular}

When a new template belongs to the same class, then the SID (relative entropy) and APD (average power difference) become zero; thus Du measure automatically becomes zero or very less.

\section{References}

[1] NIST: Advanced Encryption Standard, AES (2001), http:// csrc.nist.gov/ publications/ fips/fips-197.pdf

[2] Heijmans, H.: Morphological Image Operators. AcademiPress, San Diego (1994)

[3] Juels, A., Sudan, M.: A Fuzzy Vault Scheme. In: Lapidothand, A., Teletar, E. (eds.) proc. IEEEInt'l. Symp. Inf. Theory, p. 408 (2002)

[4] Gonzalez, R.C., Woods, R.E.: Digital Image Processing, 3rd edn. Addison-Wesley, Reading (1992) 
[5] De Mira Jr, J., Mayer, J.: Image Feature Extraction for application of Biometric Identification of Iris - A Morphological Approach. In: proc. IEEE. Int'l. Symp. on Computer Graphics and Image processing, SIBGRAPI'03.

[6] Uludag, U., Jain, A.K.: Fuzzy Finger Print Vault. In: Proc. Workshop: Biometrics: Challenges Arising from Theory to practice, pp. 13-16. W.H. Press, New York (2004)

[7] Teukolsky, S.A., Vetterling, W.T., Flannery, B.P.: Numerical Recipes in C, 2nd edn. Cambridge University press, Cambridge (1992)

[8] Teo, C.C., Ewe, H.T.: An efficient One Dimensional Fractal Analysis for Iris Recognition, WSCG'2005, January 31 Feb4, 2005, Plzen, Czech Republic (2005)

[9] LiMA,Tieniu: Efficient Iris Recognition by Characterizing Key Local Variations, IEEE trans., Image Processing (2004)

[10] Ives, R., Etter, D., Du, Y.: Iris Pattern Extraction using Bit Planes and Standard Deviations. In: IEEE conference on Signals, systems and computers (2004)

[11] Tian, Q.-C., Pan, Q., Cheng, Y.-M.: Fast algorithm and application of Hough Transform in iris segmentation. In: proceedings of third IEEE conference on machine learning and Cybernetics, Shangai, pp. 26-29 (2004)

[12] Sharma, M., Markou, M., Singh, S.: Evaluaion of Texture Methods For Image Analaysis, pattern recognition letters. 